\title{
Article
}

\section{Interpretation of the THz-THz-Raman Spectrum of Bromoform}

\author{
loan-Bogdan Magdau, Griffin J Mead, Geoffrey A. Blake, and Thomas Francis Miller
}

J. Phys. Chem. A, Just Accepted Manuscript • DOI: 10.1021/acs.jpca.9b05165 • Publication Date (Web): 22 Jul 2019

Downloaded from pubs.acs.org on July 22, 2019

\section{Just Accepted}

"Just Accepted" manuscripts have been peer-reviewed and accepted for publication. They are posted online prior to technical editing, formatting for publication and author proofing. The American Chemical Society provides "Just Accepted" as a service to the research community to expedite the dissemination of scientific material as soon as possible after acceptance. "Just Accepted" manuscripts appear in full in PDF format accompanied by an HTML abstract. "Just Accepted" manuscripts have been fully peer reviewed, but should not be considered the official version of record. They are citable by the Digital Object Identifier (DOI®). "Just Accepted" is an optional service offered to authors. Therefore, the "Just Accepted" Web site may not include all articles that will be published in the journal. After a manuscript is technically edited and formatted, it will be removed from the "Just Accepted" Web site and published as an ASAP article. Note that technical editing may introduce minor changes to the manuscript text and/or graphics which could affect content, and all legal disclaimers and ethical guidelines that apply to the journal pertain. ACS cannot be held responsible for errors or consequences arising from the use of information contained in these "Just Accepted" manuscripts. 


\title{
Interpretation of the THz-THz-Raman Spectrum of Bromoform
}

\author{
Ioan B. Magdău, ${ }^{\dagger}$ Griffin J. Mead ${ }^{\dagger}$ Geoffrey A. Blake, ${ }^{*} \dagger$ and Thomas F. Miller \\ III*,† \\ $\dagger$ Division of Chemistry $\&$ Chemical Engineering, California Institute of Technology, \\ Pasadena, California 91125, United States \\ $\ddagger$ Division of Geological \& Planetary Sciences, California Institute of Technology, Pasadena, \\ California 91125, United States \\ E-mail: gab@gps.caltech.edu; tfm@caltech.edu
}

the experimental spectrum; instead, the experimental spectrum is recovered using RDM simulations with dipole matrix parameters that are in agreement with independent $a b$ initio calculations. The fundamental interpretation of the TTR signatures in terms of coupled intramolecular vibrational modes remains unchanged from the previous work.

\section{Introduction}

Two dimensional time resolved spectroscopy encompasses techniques in which a sequence of three or four coherent laser pulses is used to measure the response of the system with respect to the time delays. The direct Fourier transform of this response corresponds to a frequency-frequency correlation function that carries rich information about the anharmonicity, coupling and non-linearity of the vibrational and electronic states. These characteristics elucidate the underlying dynamics and various energy dissipation processes, such as homogeneous and inhomogeneous broadening.

Each laser pulse in the sequence creates or destroys coherences via one of two physical processes: optical absorption $\left(1^{\text {st }}\right.$ order in the field interaction) or Raman scattering $\left(2^{n d}\right.$ order in the field interaction). These processes can take place in the visible (VIS) regime probing elec- 
tronic states, the infrared (IR) regime measuring high frequency intra-molecular vibrational modes, or the terahertz $(\mathrm{THz})$ regime probing low-frequency modes such as lattice phonons or inter-molecular vibrations. Combination of these various laser pulses leads to a range of different spectroscopic methods such as 2D-IR, 2D-VIS, VIS-IR, 2D-Raman, etc., each with its own applicability.

2D-IR spectroscopy was one of the first methods used to investigate the vibrational properties of liquids, and it has helped address topics that include structural fluctuations in water, ${ }^{1-4}$ proton shuttling in acidic solutions $^{5,6}$ and protein dynamics. ${ }^{7-9}$ While 2D-IR has proven useful for studying highfrequency vibrational modes, there is strong motivation to extend multidimensional spectroscopy to the low-frequency regime. Important physical chemical processes, such as collective solvent motions ${ }^{10-12}$ and dynamics of large biomolecules ${ }^{13-15}$ are driven by processes that takes place in the $\mathrm{THz}$ range. The first method developed to study liquids in the $\mathrm{THz}$ domain was 2D-Raman spectroscopy. ${ }^{16}$ This method is $5^{\text {th }}$ order in the laser field, and it has been shown to suffer from cascading effects where the $5^{\text {th }}$ order response is plagued by contributions from higher intensity $3^{\text {rd }}$ order processes. ${ }^{17}$ It is only with difficulty that these cascaded processes were overcome to yield the true $5^{\text {th }}$ order 2D Raman response. ${ }^{18,19}$

The advent of powerful $\mathrm{THz}$ sources ${ }^{20,21}$ has enabled $3^{\text {rd }}$ order hybrid spectroscopic techniques, in which one or two of the $2^{\text {nd }}$ order Raman processes are replaced by $1^{\text {st }}$ order THz absorption: RTT, TRT, and TTR. ${ }^{22}$ The RTT and TRT methods measure a THz emission and were first employed to study water and aqueous solutions. ${ }^{12,23-25}$ TTR builds upon the pulse-detection methods developed in $\mathrm{THz}-$ Kerr-effect spectroscopy, ${ }^{26,27}$ which has been successfully applied in both polar and non-polar liquids ${ }^{28,29}$. The TTR approach was recently developed in our group ${ }^{30,31}$ and used to measure the $3^{\text {rd }}$ order nonlinear response of bromoform ${ }^{32}$ $\left(\mathrm{CHBr}_{3}\right)$, carbon tetrachloride $\left(\mathrm{CCl}_{4}\right)$ and dibromodichloromethane $\left(\mathrm{CBr}_{2} \mathrm{Cl}_{2}\right)$. These halogen liquids are ideal test systems for TTR be- cause they exhibit heavy intra-molecular modes in the $\mathrm{THz}$ frequency regime and are Ramanactive due to their strong polarizablity. ${ }^{33}$

All three Raman-THz hybrid methods (i.e., RTT, TRT, and TTR) are complementary and measure different correlation functions involving the dipole and polarizability surfaces. These responses carry rich information about the systems under study, including the nonlinearity of the dipole and polarizability surfaces and the anharmonicity and mechanical coupling of the various vibrational motions. However, this information is encoded in complex, threepoint correlation functions that must be disentangled with the aid of theoretical and computational methods. ${ }^{30,31,34-48}$ This problem of interpretation is further complicated by the number of vibrational states that need to be considered. Whereas infrared-active modes are usually in their vibrational ground state at room temperature and typically only involve single excitations upon illumination, THz-active modes can be thermally excited at room temperature, and multiple transitions between the different states are possible.

In our previous work, ${ }^{31}$ we developed a reduced density matrix (RDM) model to understand the TTR spectrum in liquid bromoform. When the parameters for the nonlinear dipole elements in the RDM model were fit to best reproduce the experimental TTR signal, it was found that they assumed unexpectedly large values that did not agree with our accompanying $a b$ initio electronic structure calculations. Here, we reconcile this apparent inconsistency by developing a more complete description of the TTR spectra from both the theoretical and experimental perspective.

\section{Method Development}

We begin by reviewing the RDM model of Ref. 30,31. In section Interpreting the Experimental Data (), we describe the time-coordinate transformation necessary to reinterpret the experimental spectrum. In sections and, we explain the development of a new RDM model that fully accounts for the liquid symmetry and 
the symmetries of the dipole and polarizability matrices to $4^{\text {th }}$ order in the normal modes.

In $\mathrm{RDM}$, we propagate the reduced density matrix $\rho(t)$ for a single bromoform molecule by solving the Liouville-von Neumann equation, ${ }^{49}$

$$
\frac{\partial \rho(t)}{\partial t}=-\frac{i}{\hbar}[H(t), \rho(t)]-\Gamma \rho(t),
$$

where $\Gamma$ is a constant phenomenological relaxation matrix that accounts for the interaction with the bath by population relaxation $\left(\Gamma_{n n}\right.$ $=\frac{1}{\text {, where }} \tau_{\tau_{1}} \tau_{1}=100 \mathrm{fs}$ ) and coherence dephasing $\left(\Gamma_{n m}=\frac{1}{\tau_{2}}\right.$, where $\left.\tau_{2}=2 \mathrm{ps}\right)$. We tested longer population relaxation times up to $10 \mathrm{~ns}$, and we found no change in the computed TTR response.

The time-varying Hamiltonian, $H(t)$, describes interactions of the system with the $\mathrm{THz}$ fields,

$$
H\left(t ; t_{1}\right)=H-M \cdot E_{T}(t)-M \cdot E_{T}\left(t-t_{1}\right),
$$

where $E_{T}(t)$ is the experimentally measured pulse shape, ${ }^{31} H$ is the system Hamiltonian, and $M$ is the transition dipole matrix. Using $E_{T}(t)$ in the simulation, instead of a simple delta function $E \delta(t)$ ensures that the computed spectrum is in fact the molecular response convoluted with the instrument response function (IRF) and can be directly compared to the raw experimental data.

We propagate equation 1 forward in time by numerical integration, noting that the following mixed central-forward scheme provides good numerical stability:

$$
\begin{aligned}
\rho\left(t+d t ; t_{1}\right) & =\rho\left(t-d t ; t_{1}\right)-\frac{2 i}{\hbar}\left[H\left(t ; t_{1}\right), \rho\left(t ; t_{1}\right)\right] d t \\
& -2 \Gamma \rho\left(t-d t ; t_{1}\right) d t
\end{aligned}
$$

where the commutator is discretized by central difference, which preserves time reversibility, and the phenomenological dissipation is discretized by forward difference. We then calculate the TTR signal as the field emitted by the final Raman process,

$$
S^{T h}\left(t_{2} ; t_{1}\right)=\Re\left(\operatorname{Tr}\left(\Pi \cdot \rho\left(t_{2} ; t_{1}\right) P\right)\right),
$$

where $\Pi$ is the transition polarizability, another important model parameter, and $P$ is a matrix with elements

$$
P_{n m}=i, P_{m n}=-i, \text { and } P_{n n}=1 .
$$

$P$ ensures that the signal is emitted $90^{\circ}$ outof-phase with respect to the induced polarization. ${ }^{49}$

We compute the frequency response from the absolute value of the Fourier transform, normalized to its maximum value,

$$
\tilde{S}^{T h}\left(f_{2} ; f_{1}\right)=\mid \mathrm{FT}\left(S^{T h}\left(t_{2} ; t_{1}\right) \mid\right.
$$

For a given realization of parameters in $H, M$, and $P$, comparison of the simulated and experimental TTR signals is made via the logarithmic error function:

$\delta=\iint d f^{2}\left[\log \left(\tilde{S}^{E x}\left(f_{2} ; f_{1}\right)\right)-\log \left(\tilde{S}^{T h}\left(f_{2} ; f_{1}\right)\right)\right]^{2}$

We fit the RDM model by minimizing $\delta$ with respect to $H, M$, and $P$, subject to $L_{2}$-norm regularization. Specifically, we minimize

$$
\Delta=\delta+\lambda\left(h_{i}^{2}+\mu_{j}^{2}+\alpha_{k}^{2}\right),
$$

where $h_{i}, \mu_{i}$ and $\alpha_{i}$ are the parameters of the Hamiltonian $H=H\left(h_{i}\right)$, transition dipole $M=$ $M\left(\mu_{i}\right)$ and transition polarizability $\Pi=\Pi\left(\alpha_{i}\right)$ operators.

We note that Eq. 1 only accounts for Markovian dissipative processes. This description may of course be extended to include more complex relaxation phenomena such as coherence and population transfer in the context of non-Markovian dynamics, ${ }^{50-52}$ which have been shown to be important in some liquids.

\section{Interpreting the Experimental Data}

The experimental setup ${ }^{30}$ consists of two $\mathrm{THz}$ pump pulses, $\mathrm{THz}_{1}$ and $\mathrm{THz}_{2}$, which are polarized along the $\mathrm{Y}$ and $\mathrm{X}$ directions and separated by a time delay, $t_{1}=t_{\mathrm{THz}_{1}}-t_{\mathrm{THz}_{2}}$. A nearinfrared (NIR) optical probe, polarized along $\mathrm{X}$, induces a Y-polarized Raman response from 
the system at time delay $t_{2}=t_{\mathrm{NIR}}-t_{\mathrm{THz}_{1}}$. Due to the symmetry of the third-order response, a Raman signal is only detected after the second THz pump has interacted with the system. Experimentally, $t_{1}$ is scanned by changing the path length that the $\mathrm{THz}_{2}$ field travels, while the $\mathrm{THz}_{1}$ field remains fixed in time. A consequence of this design is that the third-order response will occur at $t_{2}=0$ when $\mathrm{THz}_{1}$ is the second field interaction $\left(t_{1}>0\right)$, but at a time $t_{2}=t_{1}$ when $\mathrm{THz}_{2}$ is the second interaction $\left(t_{1}<0\right)$. Qualitatively, a diagonally skewed TTR response is observed in the $t_{1}<0$ region. This must be properly accounted for in the interpretation of the spectrum.

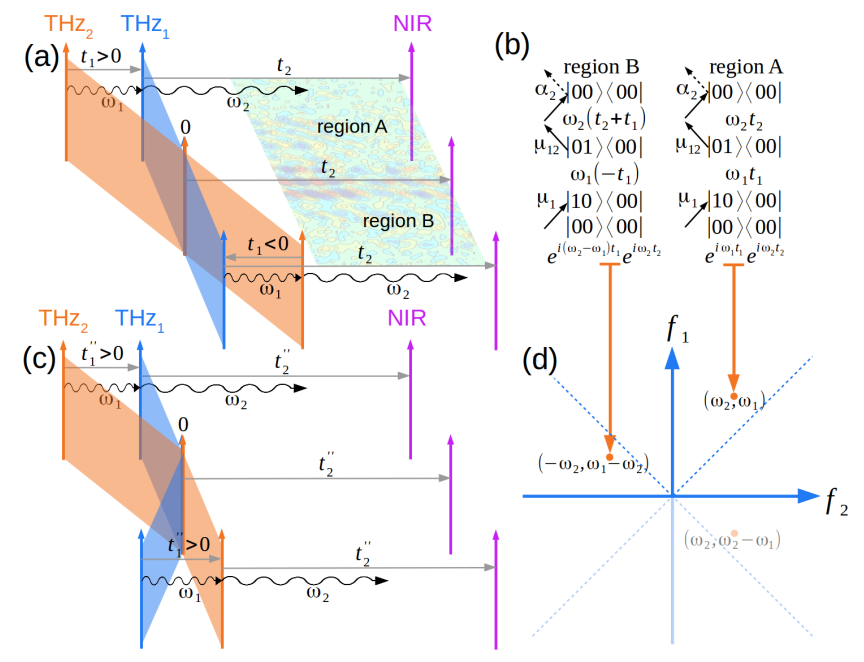

Figure 1: Illustration of the $\mathrm{THz}$ pump pulses switching time ordering and the implication on the spectral features. Panel (a) shows schematically the experimental setup and the definition of time coordinates $t_{1}$ and $t_{2}$ in the two regions of response, $\mathrm{A}$ and $\mathrm{B}$, corresponding to the pulse sequence $\mathrm{THz}_{2}-\mathrm{THz}_{1}-\mathrm{NIR}$ and $\mathrm{THz}_{1^{-}}$ $\mathrm{THz}_{2}$-NIR, respectively. Panel (b) illustrates a generic Liouville pathway represented by two distinct Feynman diagrams that feature different oscillatory phases in the portions of response $\mathrm{A}$ and $\mathrm{B}$, as a consequence of mixing the time coordinates. These two Feynman diagrams which represent the same physical process manifest as two distinct peaks in the Fourier representation of the response: panel (d). Finally, panel (c) illustrates the transformed time coordinates $t_{1}^{\prime \prime}$ and $t_{2}^{\prime \prime}$ which fix this issue.
To illustrate the importance of correctly including this skew in the analysis, we consider how a Liouville pathway in a generic system composed of two vibrational modes, changes when evaluated at $t_{1}>0$ and $t_{1}<0$. In Fig. 1(a), we label these two regions $\mathrm{A}$ and $\mathrm{B}$, corresponding to pulse ordering $\mathrm{THz}_{2}-\mathrm{THz}_{1}-$ $\operatorname{NIR}\left(t_{1}>0\right)$ and $\mathrm{THz}_{1}-\mathrm{THz}_{2}-\mathrm{NIR}\left(t_{1}<0\right)$, respectively. When the pathway is sampled in region $\mathrm{A}$ of the response, the first $\mathrm{THz}$ pulse excites coherence $|10\rangle\langle 00|$, which oscillates at frequency $\omega_{1}$ and acquires phase $e^{i \omega_{1} t_{1}}$, while the second $\mathrm{THz}$ pulse switches the coherence to $|01\rangle\langle 00|$ which oscillates at frequency $\omega_{2}$ and acquires phase $e^{i \omega_{2} t_{2}}$. Overall, this physical process generates a signal that is proportional to $e^{i \omega_{1} t_{1}} e^{i \omega_{2} t_{2}}$. The same pathway, when sampled in region $\mathrm{B}$, generates a signal that is proportional to $e^{-i \omega_{1} t_{1}} e^{i \omega_{2}\left(t_{2}+t_{1}\right)}$, or rearranging, $e^{i\left(\omega_{2}-\omega_{1}\right) t_{1}} e^{i \omega_{2} t_{2}}$. Hence, taking the Fourier transform of the full $t_{1}$ domain (including both regions $\mathrm{A}$ and $\mathrm{B}$ ) results in two distinct peaks at $\left(\omega_{2}, \omega_{1}\right)$ and $\left(\omega_{2}, \omega_{2}-\omega_{1}\right)=\left(-\omega_{2}, \omega_{1}-\omega_{2}\right)$ that otherwise represent the same physical process. This is undesirable and creates the opportunity for misinterpreting the TTR spectrum.

To ensure that each physical process is represented by a single distinct peak, we perform a coordinate transformation of the time response before computing the frequency response by FT. This transformation consists of skewing the response along $t_{2}$,

$$
\left(t_{2}^{\prime}, t_{1}^{\prime}\right)= \begin{cases}\left(t_{2}, t_{1}\right), & \text { region } \mathrm{A} \\ \left(t_{2}+t_{1}, t_{1}\right), & \text { region } \mathrm{B}\end{cases}
$$

and then flipping it with respect to $t_{1}$,

$$
\left(t_{2}^{\prime \prime}, t_{1}^{\prime \prime}\right)= \begin{cases}\left(t_{2}^{\prime}, t_{1}^{\prime}\right)=\left(t_{2}, t_{1}\right), & \text { region } \mathrm{A} \\ \left(t_{2}^{\prime},-t_{1}^{\prime}\right)=\left(t_{2}+t_{1},-t_{1}\right), & \text { region } \mathrm{B}\end{cases}
$$

as shown in Fig. 1(c). With these transformed time coordinates, our illustrative Liouville pathway acquires the same phase factors $e^{i \omega_{1} t_{1}^{\prime \prime}} e^{i \omega_{2} t_{2}^{\prime \prime}}$ when sampled in region $\mathrm{B}$ as in region $\mathrm{A}$. This transformation simplifies the resulting TTR spectrum and eliminates the appearance of redundant peaks. 


\section{Enforcing Inversion Symmetry in the RDM Model}

Liquids are isotopic, such that any response function,

$$
S(E)=\varepsilon_{1} E+\varepsilon_{2} E^{2}+\varepsilon_{3} E^{3}+\varepsilon_{4} E^{4}+\ldots,
$$

must obey inversion symmetry with respect to an applied electric field, $E$. The TTR response must also obey this symmetry,

$$
S\left(-E_{T},-E_{T},-E_{N I R}\right)=-S\left(E_{T}, E_{T}, E_{N I R}\right),
$$

and requires that even-order contributions to the experimentally observed response must vanish,

$$
\varepsilon_{2}=\varepsilon_{4}=\ldots=0 .
$$

This means that only scattering processes that involve an odd number of photons, not counting the final emission, can generate an experimental TTR signal. In the case of liquid bromoform, the final Raman process is linear and it only involves a one-photon absorption. ${ }^{31}$ Therefore, the total number of $\mathrm{THz}$ photon interactions must be even and the leading contribution to the TTR signal is $3^{\text {rd }}$ in the field, involving the absorption of two $\mathrm{THz}$ photons and one NIR photon. In other words, the TTR response scales linearly with each of the $\mathrm{THz}$ fields.

Fig. 2(b) lists all possible $3^{\text {rd }}$ order interactions in which two $\mathrm{THz}$ photons are involved: 1-1-1, 2-0-1 and 0-2-1. The TTR experiment further isolates the desired 1-1-1 response by employing differential chopping. ${ }^{30}$ In the RDM simulations, we implement differential chopping by separating the $1 \mathrm{D}$ responses $S_{2}^{T h}$ and $S_{1}^{T h}$ from the total response $S^{T h}$, as illustrated in Fig. 2(a). We compute these individual responses by propagating the dynamics under the partial Hamiltonians

$$
\begin{aligned}
& H_{2}\left(t ; t_{1}\right)=H-M \cdot E_{T}(t) \text { and } \\
& H_{1}\left(t ; t_{1}\right)=H-M \cdot E_{T}\left(t-t_{1}\right) .
\end{aligned}
$$

This simulation procedure removes the unwanted field interactions, shaded purple in Fig. 2, from the $3^{\text {rd }}$ response. However, the RDM simulated response ${ }^{30,31}$ also includes $4^{\text {th }}$ order interactions, unlike the experiment where even order processes vanish due to the symmetry of the liquid, as explained above.

It is found that for the simulated TTR response, $4^{\text {th }}$ order contributions can be comparable in magnitude to $3^{\text {rd }}$ order ones, because the latter require at least one nonlinear dipole interaction $^{\text {ii }}$ while the former do not. For instance, in Fig. 2(e), we compare two similar pathways of $3^{\text {rd }}$ and $4^{\text {th }}$ order. In the $3^{\text {rd }}$ order process, the two-quanta excitation from $|10\rangle$ to $|01\rangle$ is achieved by a single photon interacting with a nonlinear dipole element and has a scattering amplitude proportional to

$$
\sigma_{3^{r d}} \propto\left\langle 10\left|\frac{\partial^{2} M}{\partial Q_{1} \partial Q_{2}}\right| 01\right\rangle E_{T} .
$$

Meanwhile, in the $4^{\text {th }}$ order process, the same excitation is achieved by two consecutive photons acting on the linear dipole via an intermediate state,

$$
\sigma_{4^{t h}} \propto\left\langle 10\left|\frac{\partial M}{\partial Q_{2}}\right| 11\right\rangle\left\langle 11\left|\frac{\partial M}{\partial Q_{1}}\right| 01\right\rangle E_{T}^{2} .
$$

For large THz fields, this "all-linear" $4^{\text {th }}$ order can become larger than the $3^{\text {rd }}$ order if the system has small dipole nonlinearities, i.e.,

$$
\frac{\partial^{2} M}{\partial Q_{1} \partial Q_{2}} \lesssim E_{T} \frac{\partial M}{\partial Q_{2}} \frac{\partial M}{\partial Q_{1}} .
$$

To address this issue, $4^{\text {th }}$ order contributions to the simulated RDM response must be explic-

\footnotetext{
${ }^{\text {ii }}$ To prove that all $3^{\text {rd }}$ order TTR pathways require a nonlinear dipole interaction, consider a $3^{\text {rd }}$ order Liouville pathway which starts from a generic population state $|a\rangle\langle a|$. Interaction of the system with the first $\mathrm{THz}$ pulse changes the excitation state of the bra or ket of the density matrix by $n$ quanta to a $|a+n\rangle\langle a|$ coherence. Then, upon a second $\mathrm{THz}$ interaction, the system changes to either $|a+n+m\rangle\langle a|$ or $|a+n\rangle\langle a+m|$. Since the final Raman interaction only changes the system by a single quantum, ${ }^{31}$ and since all pathways must end in a population state, it follows that either $|n+m|=1$ or $|n-m|=1$. All paths involving $n=0$ or $m=0$ are removed by differential chopping, and therefore all surviving diagrams have both nonzero $n$ and $m$. As a result, at least one of the $\mathrm{THz}$ processes exchanges more than one quantum with the systems, and it is strictly nonlinear.
} 
(b) $3^{\text {rd }}$ order interactions

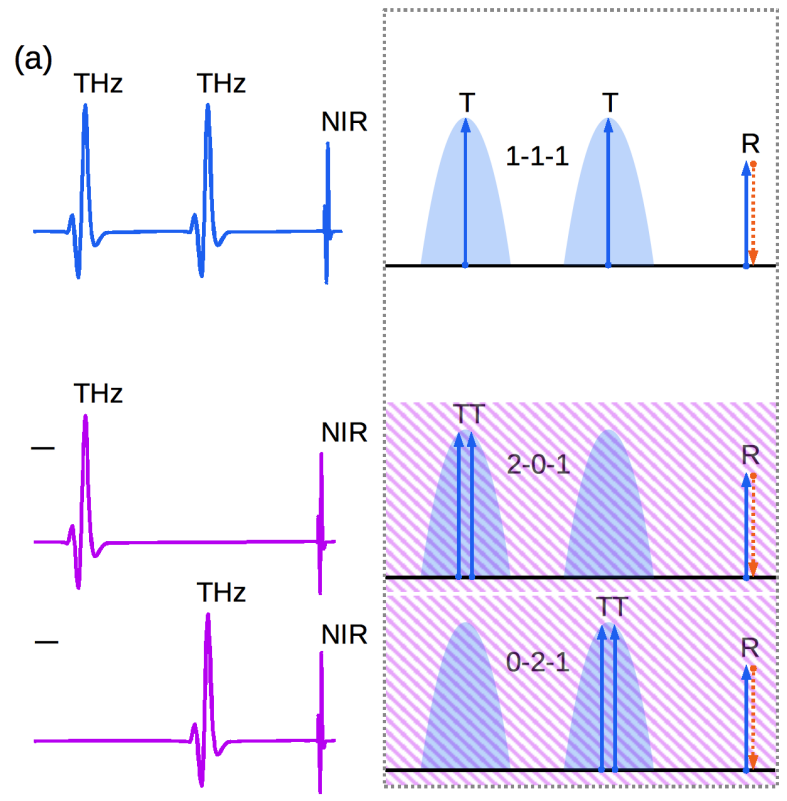

(c) $4^{\text {th }}$ order interactions

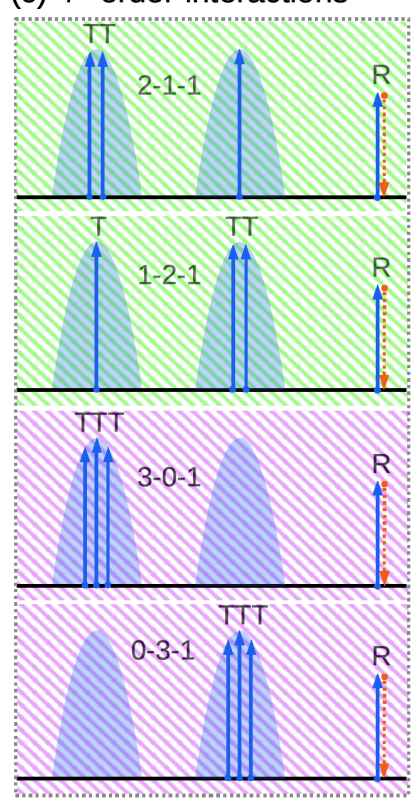

(d)
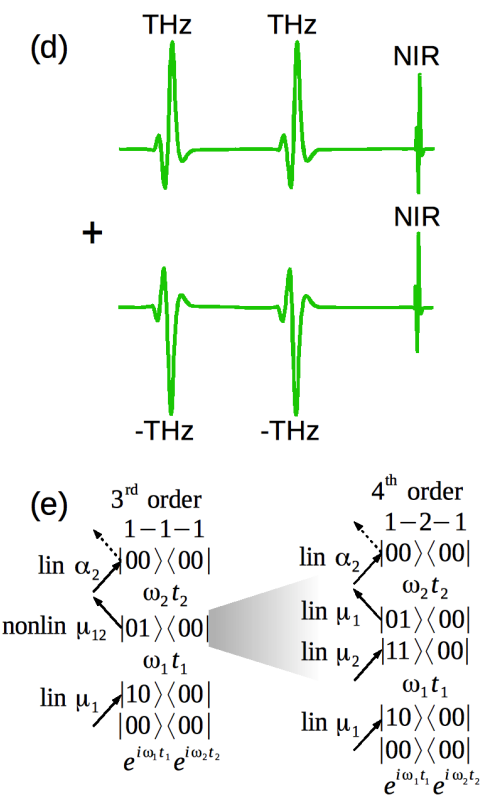

Figure 2: Differential chopping and enforced inversion symmetry remove unwanted responses from the spectrum generated via RDM. Panel (a) schematically illustrates differential chopping, while panel (d) shows how we enforce inversion symmetry. Panels (b) and (c) list all $3^{\text {rd }}$ and $4^{\text {th }}$ order field interactions manifested in the RDM, from which only the 1-1-1 process is sampled experimentally. Shaded in purple are the processes removed by differential chopping, and in green the processes removed by enforcing inversion symmetry. Panel (e) exemplifies two similar Liouville pathways of $3^{\text {rd }}$ and $4^{\text {th }}$ order, in which the transfer of coherence from $|10\rangle\langle 00|$ to $|01\rangle\langle 00|$ takes place via one nonlinear dipole interaction in the former and two linear dipole interactions in the latter.

itly removed. We achieve this by simulating both the response to positive $\mathrm{THz}$ fields and negative $\mathrm{THz}$ fields, and then summing the two contributions as illustrated in Fig. 2(d),

$$
S^{T h}=S^{T h}\left(E_{T}, E_{T}\right)+S^{T h}\left(-E_{T},-E_{T}\right) .
$$

This procedure ensures that the final response function is odd with respect to the overall field and that the $4^{\text {th }}$ order contributions, shaded green in Fig. 2(c), vanish. As a result, the simulated TTR spectrum correctly reflects the inversion symmetry of the liquid state and can be compared directly to the experimental spectrum.

\section{The Hamiltonian, Dipole, and Po- larizability Surfaces}

The bromoform molecule has three vibrational degrees of freedom in the terahertz regime: two degenerate $\mathrm{C}-\mathrm{Br}$ bending modes at $4.7 \mathrm{THz}$,
$Q_{1}$ and $Q_{2}$, and a symmetric umbrella mode at $6.6 \mathrm{THz}, Q_{3} .{ }^{53}$ Fig. 3(a) illustrates these motions and provides an energy-level diagram with the number of quanta in each mode indicated, using notation $\left|Q_{1} Q_{2} Q_{3}\right\rangle$. In typical 2DIR spectroscopy applications at room temperature, only the ground and first-excited state of each mode are involved. TTR spectroscopy probes modes that are low in energy, such that even at room temperature $(k T \approx 6.2 \mathrm{THz})$, it is necessary to consider thermally accessible excited states. In the current study, the bandwidth of the $\mathrm{THz}$ pulse covers $B W \approx 8$ $\mathrm{THz}$ of energy, such that all the states below $k T+B W \lesssim 14.2 \mathrm{THz}$ should be considered. The highest energy state that is included in our model is the quadruplet $Q_{1} \otimes Q_{2}$, amounting to three quanta of energy in the degenerate mode $(3 \times 4.7 \mathrm{THz}=14.1 \mathrm{THz})$.

The calculated TTR spectrum depends on the parameterization of $H=H\left(h_{i}\right), M=M\left(\mu_{i}\right)$ and $\Pi=\Pi\left(\alpha_{i}\right)$. In the RDM model of Ref. 31, 
we employed a harmonic Hamiltonian,

$$
\hat{H}=\hbar \omega_{1}\left(\hat{a}_{1}^{\dagger} \hat{a}_{1}+\hat{a}_{2}^{\dagger} \hat{a}_{2}\right)+\hbar \omega_{3} \hat{a}_{3}^{\dagger} \hat{a}_{3},
$$

the parameters for which were fixed to the linear absorption experimental spectrum, and a transition dipole of the form

$$
\begin{aligned}
\hat{M} & =\mu_{1}\left(\hat{Q}_{1}+\hat{Q}_{2}\right)+\mu_{2} \hat{Q}_{3}+\hat{M}_{2 q}\left(\mu_{4}, \mu_{4}^{\prime}, \mu_{4}^{\prime \prime}\right) \\
& +\hat{M}_{3 q}\left(\mu_{7}, \mu_{7}^{\prime}, \mu_{8}\right)+\hat{M}_{4 q}\left(\mu_{11}, \mu_{12}\right)
\end{aligned}
$$

where $\hat{M}_{n q}$ represent the nonlinear blocks of the dipole operator comprised of $n$-quanta $(n>$ 1) transitions, which were determined by fitting to the nonlinear TTR experimental spectrum. The $\hat{Q}_{i}$ are non-dimensional normal modes which can be expressed in terms of the creation and annihilation operators $\hat{a}_{i}^{\dagger}$ and $\hat{a}_{i}$,

$$
\hat{Q}_{i}=\sqrt{\frac{2 m_{i} \omega_{i}}{\hbar}} \hat{q}_{i}=\hat{a}_{i}^{\dagger}+\hat{a}_{i} .
$$

Combining Eqs. 21 and 20 and integrating the linear part of the dipole in the $\left|Q_{1} Q_{2} Q_{2}\right\rangle$ basis leads to the matrix parameterization of Ref. 31 (Fig. 3(b)).

In this work, we develop a more comprehensive model for the Hamiltonian, which includes the anharmonicity $\Delta_{i}$ of the single molecule normal modes, as well as mode coupling $\beta_{i j}$ originating from the condensed-phase environment. We employ a quantum-conserving Hamiltonian of the form

$$
\begin{aligned}
\hat{H} & =\hbar \omega_{1}\left(\hat{a}_{1}^{\dagger} \hat{a}_{1}+\hat{a}_{2}^{\dagger} \hat{a}_{2}\right)+\hbar \omega_{3} \hat{a}_{3}^{\dagger} \hat{a}_{3}+\beta_{12}\left(\hat{a}_{1}^{\dagger} \hat{a}_{2}+\hat{a}_{1} \hat{a}_{2}^{\dagger}\right)+\beta_{13}\left(\hat{a}_{2}^{\dagger} \hat{a}_{3}+\hat{a}_{2} \hat{a}_{3}^{\dagger}+\hat{a}_{1}^{\dagger} \hat{a}_{3}+\hat{a}_{1} \hat{a}_{3}^{\dagger}\right) \\
& +\Delta_{1}\left(\hat{a}_{1}^{\dagger 2} \hat{a}_{1}^{2}+\hat{a}_{2}^{\dagger 2} \hat{a}_{2}^{2}\right)+\Delta_{3} \hat{a}_{3}^{\dagger \dagger} \hat{a}_{3}^{2},
\end{aligned}
$$

and integrate it in the $\left|Q_{1} Q_{2} Q_{2}\right\rangle$ basis to obtain the matrix representation shown in Fig. 3(c). Note that the couplings between the doubly degenerate modes $Q_{1,2}$ and mode $Q_{3}$ are equivalent and labeled $\beta_{13}$ in Eq. 22.
Furthermore, we Taylor expand the dipole operator to $4^{\text {th }}$ order in the normal modes and enforce the $Q_{1,2}$ symmetry by grouping together terms which are invariant to label exchange $(1 \leftrightarrow 2)$ :

$$
\begin{aligned}
\hat{M} & =\mu_{1}\left(\hat{Q}_{1}+\hat{Q}_{2}\right)+\mu_{2} \hat{Q}_{3}+\frac{1}{2 !} \mu_{3}\left(\hat{Q}_{1}^{2}+\hat{Q}_{2}^{2}\right)+\mu_{3}^{*} \hat{Q}_{1} \hat{Q}_{2}+\mu_{4}\left(\hat{Q}_{1}+\hat{Q}_{2}\right) \hat{Q}_{3}+\frac{1}{2 !} \mu_{5} \hat{Q}_{3}^{2} \\
& +\frac{1}{3 !} \mu_{6}\left(\hat{Q}_{1}^{3}+\hat{Q}_{2}^{3}\right)+\frac{1}{2 !} \mu_{6}^{*} \hat{Q}_{1} \hat{Q}_{2}\left(\hat{Q}_{1}+\hat{Q}_{2}\right)+\frac{1}{2 !} \mu_{7}\left(\hat{Q}_{1}^{2}+\hat{Q}_{2}^{2}\right) \hat{Q}_{3}+\mu_{7}^{*} \hat{Q}_{1} \hat{Q}_{2} \hat{Q}_{3}+\frac{1}{2 !} \mu_{8}\left(\hat{Q}_{1}+\hat{Q}_{2}\right) \hat{Q}_{3}^{2} \\
& +\frac{1}{3 !} \mu_{10}^{*} \hat{Q}_{1} \hat{Q}_{2}\left(\hat{Q}_{1}^{2}+\hat{Q}_{2}^{2}\right)+\frac{1}{2 ! 2 !} \mu_{10}^{* *} \hat{Q}_{1}^{2} \hat{Q}_{2}^{2}+\frac{1}{3 !} \mu_{11}\left(\hat{Q}_{1}^{3}+\hat{Q}_{2}^{3}\right) \hat{Q}_{3}+\frac{1}{2 !} \mu_{11}^{*} \hat{Q}_{1} \hat{Q}_{2}\left(\hat{Q}_{1}+\hat{Q}_{2}\right) \hat{Q}_{3} \\
& +\frac{1}{2 ! 2 !} \mu_{12}\left(\hat{Q}_{1}^{2}+\hat{Q}_{2}^{2}\right) \hat{Q}_{3}^{2}+\frac{1}{2 !} \mu_{12}^{*} \hat{Q}_{1} \hat{Q}_{2} \hat{Q}_{3}^{2}
\end{aligned}
$$

Whereas the dipole form in Ref. 31 (Eq. 20) uses a normal mode expansion of the linear part and an empirical parameterization of the nonlinear part, the current work expands the full dipole operator in the normal modes to $4^{\text {th }}$ order. As a result, the parameters of the current model are associated with the partial deriva- tives of $\hat{M}$ with respect to the $\hat{Q}_{i}$. We express the $n^{\text {th }}$ power of $\hat{Q}_{i}$ as creation-annihilation $n$ - 

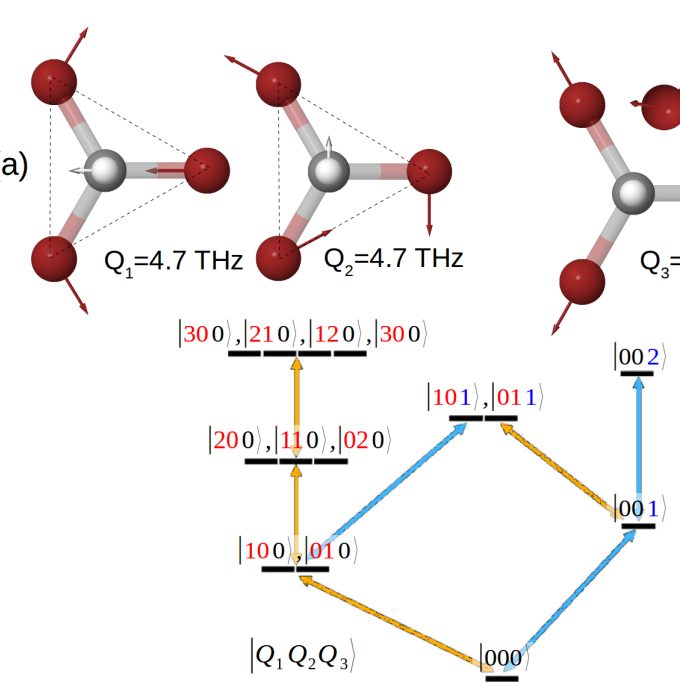

(c)

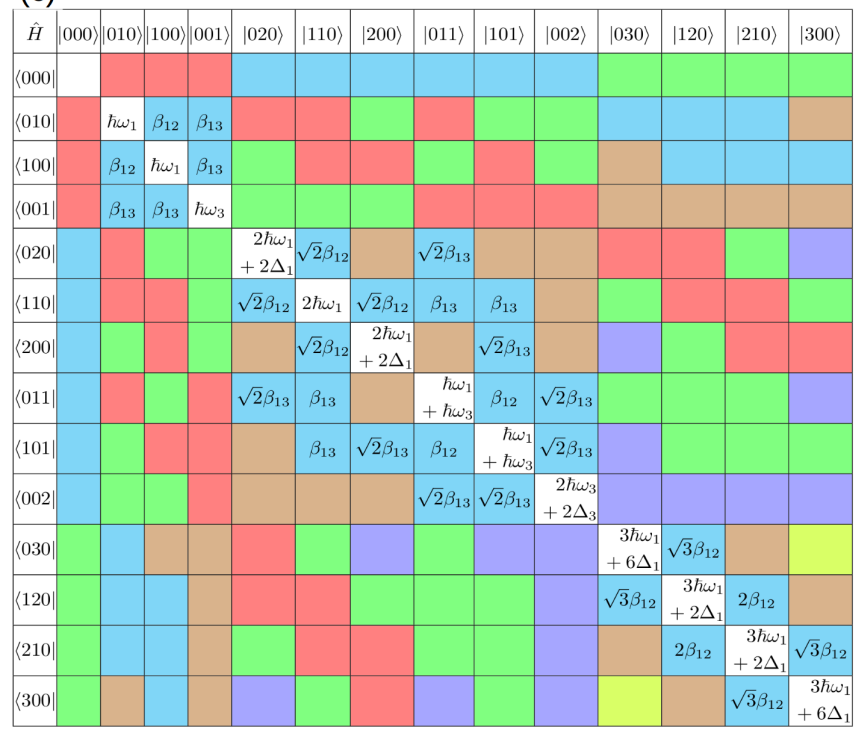

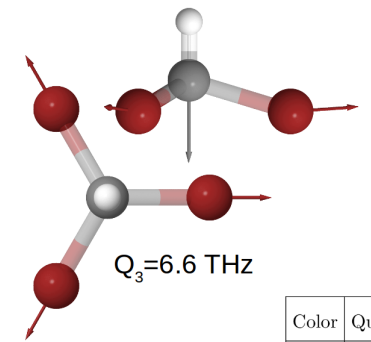

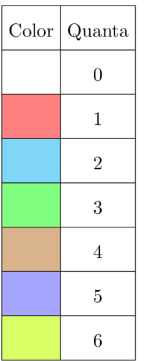

(d)

(b)
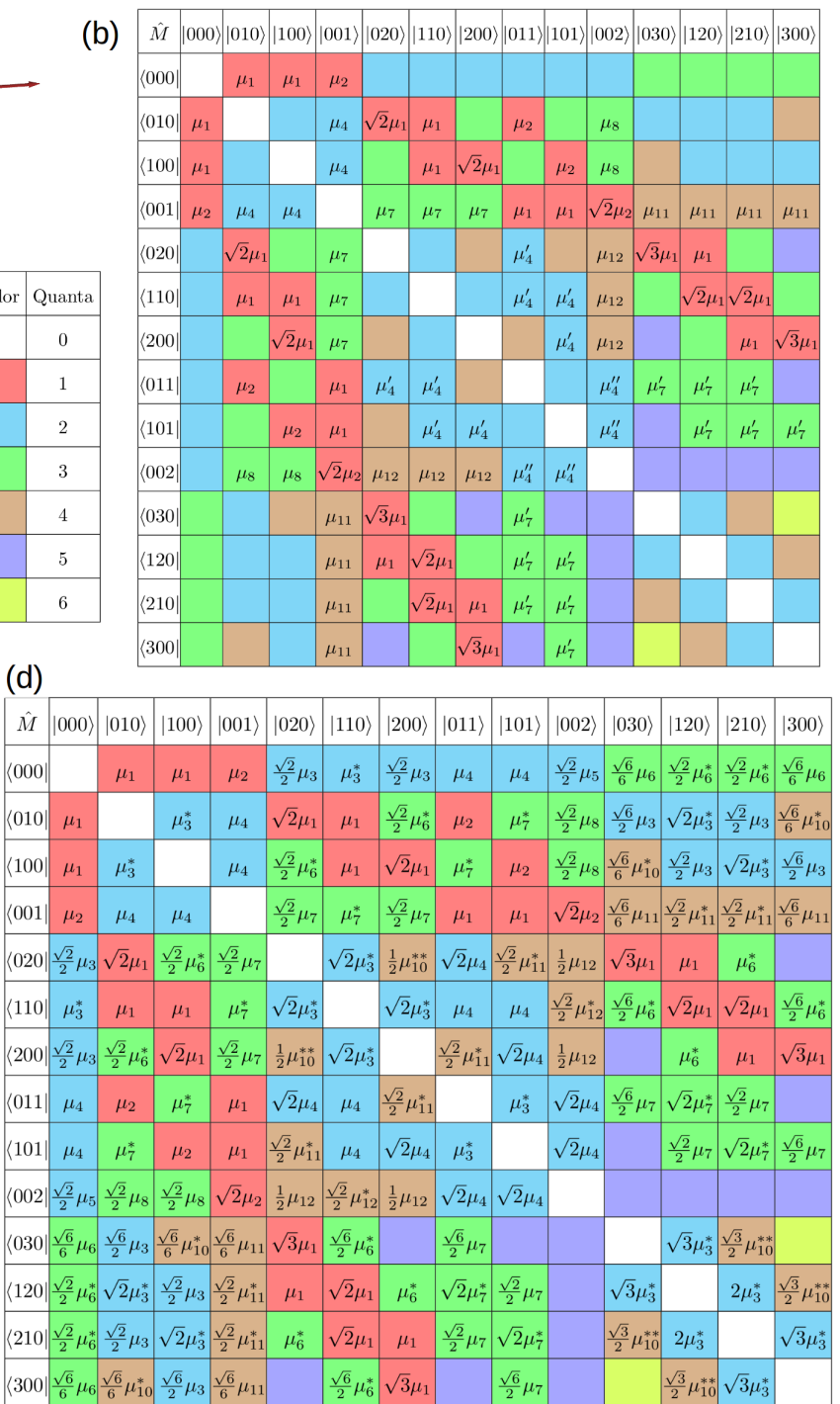

Figure 3: Panel (a) shows the low energy normal modes of the bromoform molecule: the degenerate "C-Br bending modes" $Q_{1}$ and $Q_{2}$ at $4.7 \mathrm{THz}$ and the "umbrella mode" $Q_{3}$ at $6.6 \mathrm{THz}$. The panel also shows the corresponding quantum states labeled $\left|Q_{1} Q_{2} Q_{3}\right\rangle$ that we use to describe these normal modes. The double arrow indicates all possible 1-quantum transitions between these states. Panel (b) shows the parametrization of the transition dipole moment $(\hat{M})$ used in Ref. 31. The color assigned to each matrix element corresponds to the number of quanta involved in that transition as summarized in the inset table. Elements that appear empty are fixed to zero. Panels (c) and (d) illustrate the Hamiltonian $(\hat{H})$ and transition dipole $(\hat{M})$ in the current RDM model, as derived by integrating equations 22 and 23. Notice that in this work the dipole is fully parameterized to the $4^{\text {th }}$ order and only transition matrix elements higher than $4^{\text {th }}$ order are set to zero.

tuples,

$$
\begin{aligned}
\hat{Q}_{i}^{n} & =\hat{a}_{i}^{\dagger n}+\sum_{\text {perm }} \hat{a}_{i}^{\dagger(n-1)} \hat{a}_{i}+\sum_{\text {perm }} \hat{a}_{i}^{\dagger(n-2)} \hat{a}_{i}^{2} \\
& +\ldots+\sum_{\text {perm }} \hat{a}_{i}^{\dagger 2} \hat{a}_{i}^{(n-2)}+\sum_{\text {perm }} \hat{a}_{i}^{\dagger} \hat{a}_{i}^{(n-1)}+\hat{a}_{i}^{n}
\end{aligned}
$$

and pure annihilation (last term); all other terms account for transitions involving less than $n$ quanta. We then integrate Eq. 23 in the $\left|Q_{1} Q_{2} Q_{2}\right\rangle$ basis and obtain the model illustrated in Fig. 3(d). The polarizability operator is parameterized in a similar way; however, it is only expanded to second power in $Q_{i}$, since the final Raman interaction is expected to be

and we keep only the pure creation (first term) 
linear. ${ }^{31}$

To fit the simulated RDM spectrum to the experimental TTR result, we keep $\hbar \omega_{1}, \hbar \omega_{3}, \mu_{1}$, $\mu_{3}$, and $\alpha_{1}$ fixed, while varying the remaining parameters. We perform the RDM dynamics in the diagonal representation of the Hamiltonian. As such, we diagonalize $H$,

$$
H^{\prime}=D^{\dagger} H D
$$

and apply the same transformation to the dipole and polarizability,

$$
M^{\prime}=D^{\dagger} M D, \quad \Pi^{\prime}=D^{\dagger} \Pi D
$$

performing the RDM simulations with $H^{\prime}, M^{\prime}$ and $P^{\prime}$.

\section{Results and Discussions}

The results are presented in two parts. We first present the revised experimental spectrum obtained through the transformation of the time coordinates. We then discuss the results of the $\mathrm{RDM}$ model introduced in this work.

\section{Revised Experimental Spectrum}

The raw experimental TTR response is comprised of an orientational molecular response with superimposed oscillations arising from vibrational coherences. As in previous work, ${ }^{27,30}$ we focus on the vibrational component of the response, so we use an exponential fit along $t_{2}$ to de-trend the orientational response from the data.

In Figs. 4(a)-(c), we demonstrate how the vibrational signal changes upon application of the time-coordinate transformation from section Interpreting the Experimental Data (). The first step of the transformation involves a skew along $t_{2}$, which makes the response function appear symmetric with respect to the $t_{1}=0$ line. The transformed signal is symmetric because swapping the order of the $\mathrm{THz}$ pulses does not change the response type; in both cases, we measure a TTR signal. This is unlike a TRT experiment where inverting the pulse order results in a RTT sequence which measures a different correlation function altogether. ${ }^{23}$ The second time transformation involves a flip with respect to $t_{1}$, which due to the symmetry of the response about $t_{1}=0$ simply averages the $\mathrm{A}$ and $\mathrm{B}$ regions of the data and improves the signal to noise ratio.

Panels (d) and (e) of Fig. 4 show the absolute value Fourier transforms of the original and transformed time responses, respectively. The revised spectrum is significantly simplified (panel (e)). Whereas the original spectrum features six intense peaks, the revised spectrum shows three features that are familiar in the context of conventional two dimensional spectroscopy. The most intense peak forms at $f_{1}=\omega_{3}-\omega_{1}$ and reports on the coupling between modes $Q_{1}$ and $Q_{3}$ via a nonlinear dipole interaction. The equivalent mode in the negative $f_{2}<0$ quadrant is closely related and shows a clear rephasing pathway. Note that in the revised spectrum, the $f_{2}<0$ quadrant corresponds to true rephasing, whereas in the original spectrum the peaks involve a mix of rephasing and higher overtone non-rephasing pathways. Interestingly, the most intense truerephasing feature appears tilted at an angle; this tilt could be related to the degree of inhomogeneous broadening. Finally, in the nonrephasing quadrant along the first diagonal, the higher frequency features appear symmetric to those at lower frequency.

\section{Parameterization of the RDM Model}

We fit the parameters of the RDM model with respect to the revised experimental spectrum using the Levenberg-Marquardt algorithm implemented in Octave, ${ }^{54-56}$ minimizing the penalty function in equation 8 . For each value of the regularization parameter $\lambda$, we start with random initial guesses for the RDM parameters, sampled uniformly from the $[-1,1]$ interval. The fitting problem is highly underdetermined, featuring multiple local minima, due to the large number (order $10^{4}$ ) of Liouville paths that compete in the creation of a relatively small number of peaks. Additionally, the dipole and polarizability parameters can take 


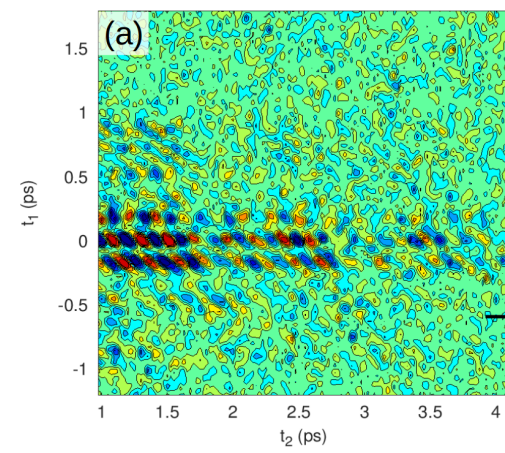

(b)

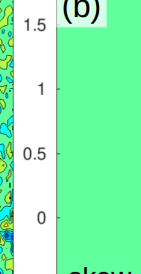

-0.5 skew
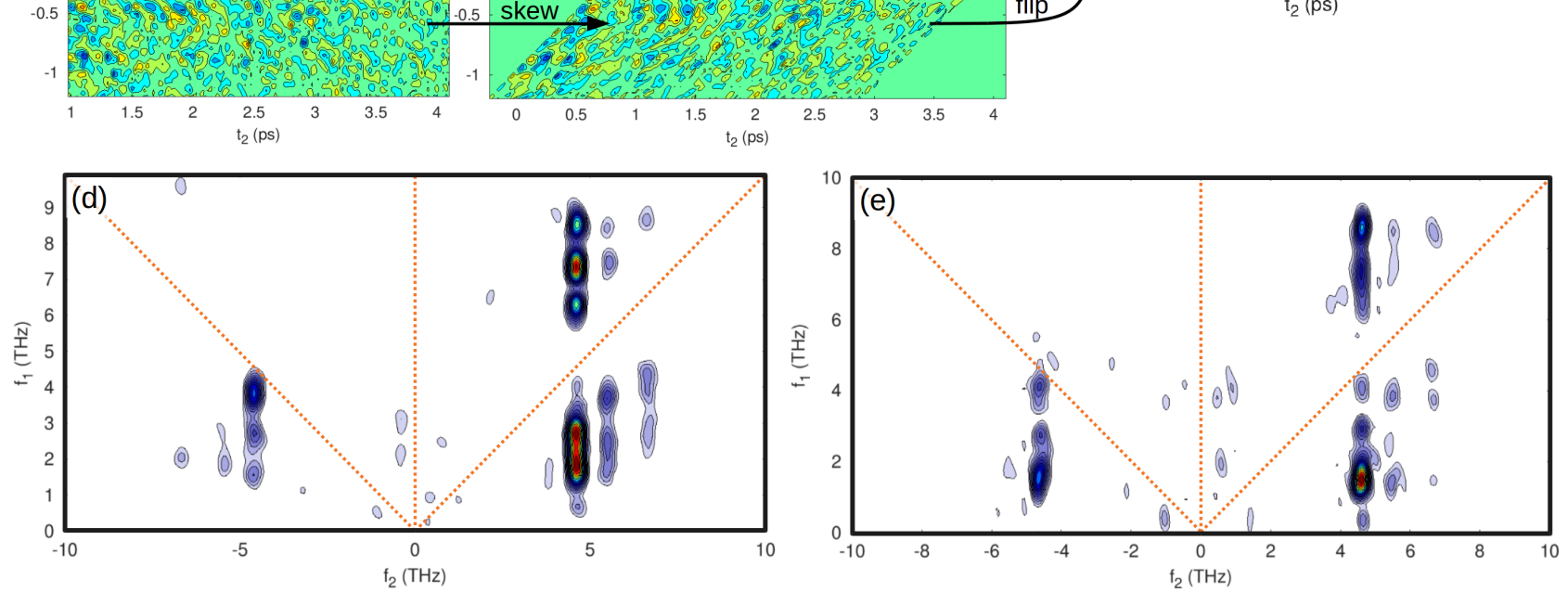

Figure 4: Illustration of the effect of the introduced time-coordinate transformation on the experimental spectrum. Panels (a), (b) and (c) show how the time transformation (skew and flip) alters the response in time domain. Panel (a) corresponds to the initial coordinates represented schematically in Fig. 1(a), while panel (c) to the proposed coordinates represented in Fig. 1(c). Panels (d) and (e) present the Fourier transforms of panels (a) and (c), before and after the time transformation, respectively.

negative values, which lead to peak cancellations. To address these challenges, we perform multiple minimizations, starting each time from a different random initial guess.

In Fig. 5(a) we plot all the converged solutions as function of the maximum fitted parameter $\max \left(h_{i}, \mu_{j}, \alpha_{k}\right)$, and color them according to the $L_{2}$ regularization strength, $\lambda$. Clustered to the left are under-fitted results with small fitting parameters but large errors, while to the right are the over-fitted results that match the spectrum at the expense of unphysical parameters. The optimal solution lies in the intermediate regime with $\lambda=100$, as indicated in Fig. 5(a). Three independent minimizations starting from different initial guesses converged to the same error with very similar parameters, suggesting relative robustness of this optimal paremeterization. We summarize the best-fit parameters in tables 1 and 2, which report the values of the transition dipole elements, and we show the full transition dipole matrix in
Fig. 5(b).

The resulting RDM simulated spectrum shown in Fig. 5(c) is in excellent agreement with the revised experimental spectrum (Fig. 4(e)). This can be clearly seen in Fig. 5(d), which provides a side-by-side comparison between simulation and experiment along the three most important 1D traces from the 2D response.

It is found that use of the experimental pulse shape $E_{T}(t)$ in the RDM simulations is crucial for reproducing the experimental spectrum. Using simple $\delta$-functions to simulate the response, otherwise with the same RDM parameters, gives a radically different result, with just one dominant peak on the diagonal at $f_{2}=4.7$ THz.

The dipole nonlinearities fitted with the RDM model in this work are significantly smaller than those found in Ref. 31, and they agree well with results from ab initio electronic structure calculations (see table 1). Critically, the third-order fitted non-linearities in the current RDM model 

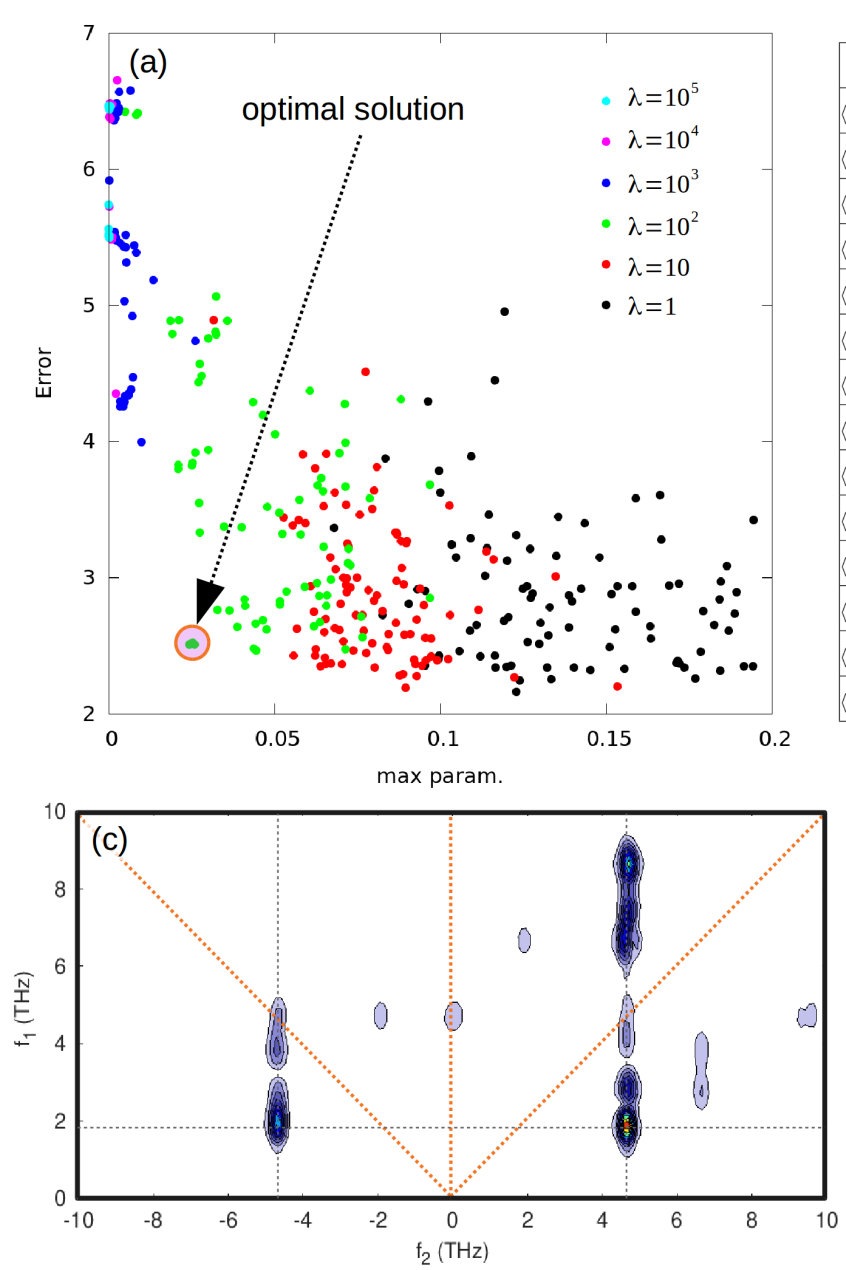

(b)

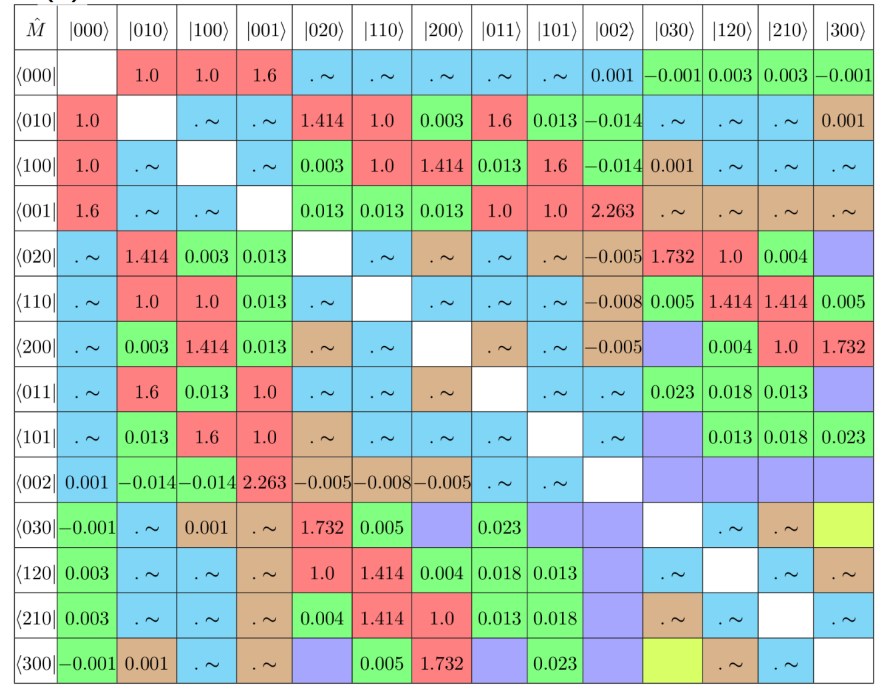

(d)

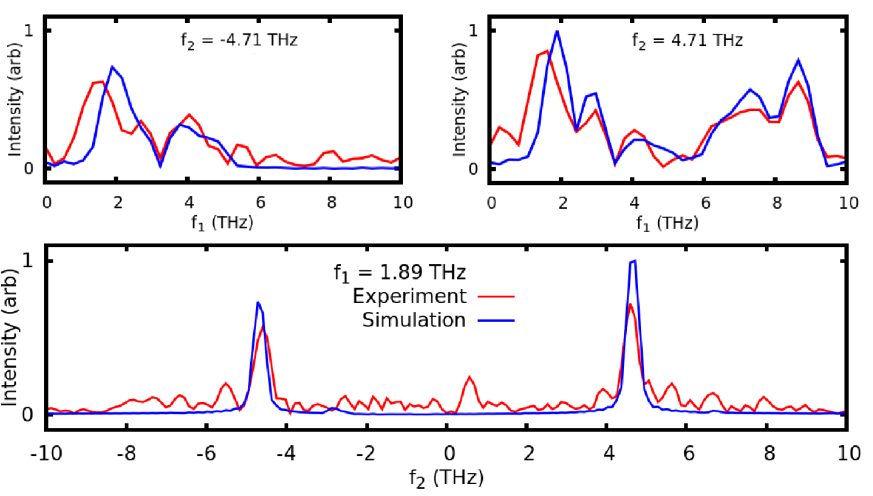

Figure 5: Fitting the parameters of the RDM model to experiment. Panel (a) illustrates the regularization procedure. Each point is a local solution represented by a set of fitted parameters $p_{i}$. Here we plot the error of each solution $\Delta E_{i}$ as function of the maximum parameter value, $\max \left(p_{i}\right)$. The color represents the different regularization coefficient $\lambda$. The optimal solution minimizes the error with the smallest possible parameters. Panels (b) and (c) show the transition dipole matrix, the simulated spectrum corresponding to the optimal solution identified in panel (a). In panel (b) we use the placeholder ". $\sim$ " for parameters that are essentially zero within three significant digits. Panel (d) shows three important 1D slices through the $2 \mathrm{D}$ spectrum at $f_{1}=1.89 \mathrm{THz}$ and $f_{2}=+-4.71 \mathrm{THz}$ in order to facilitate the comparison to the experimental result. The fit was performed with respect to the revised experimental spectrum shown in Fig. 4(d).

have the same order of magnitude as those from the $a b$ initio electronic structure calculations, in contrast with the third-order terms from the fitted RDM model of Ref. 31. This central result demonstrates that the refined treatment of the RDM model and the experimental spectrum in the current work removes the central inconsistency of Ref. 31. Likewise, the fourth-order terms in the fitted RMD model of the current work are vastly smaller than those that were necessarily invoke in the previous study. ${ }^{31}$ For the second-order terms, both the current and earlier RDM models have very small fitted values. It is found that the difference between the fitted and $a b$ initio values for the second-order terms is insignificant. We confirmed this by running the current RDM model with the $a b$ initio values for $\mu_{4}$ and all other fitted parameters unchanged, and finding that the resulting simulated spectrum is unaffected.

Finally, in Table 2, we report the fit RDM parameters for the anharmonicity $\Delta_{i}$ of the nor- 
Table 1: Values of the transition dipole matrix, obtained from $a b$ initio (CCSD/augcc-pVTZ) calculations ${ }^{31}$ and via fitting of the RDM models in Ref. 31 and in the current work.

\begin{tabular}{cc|c|c|c}
\hline \hline$\langle i|\hat{M}| j\rangle$ & $\# \mathrm{Q}$ & CCSD $^{31}$ & RDM (Ref. 31) & RDM (current) \\
\hline$|000\rangle \rightarrow|100\rangle$ & 1 & 1.00 & 1.00 (fixed) & 1.00 (fixed) \\
$|000\rangle \rightarrow|001\rangle$ & 1 & 1.01 & 1.60 (fixed) & 1.60 (fixed) \\
\hline$|100\rangle \rightarrow|001\rangle$ & 2 & 0.04 & $0.009\left(\mu_{4}\right)$ & $0.000\left(\mu_{4}\right)$ \\
$|200\rangle \rightarrow|101\rangle$ & 2 & 0.06 & $0.000\left(\mu_{4}^{\prime}\right)$ & $0.000\left(\sqrt{2} \mu_{4}\right)$ \\
$|101\rangle \rightarrow|002\rangle$ & 2 & 0.06 & $0.000\left(\mu_{4}^{\prime \prime}\right)$ & $0.000\left(\sqrt{2} \mu_{4}\right)$ \\
\hline$|100\rangle \rightarrow|002\rangle$ & 3 & 0.00 & $0.364\left(\mu_{8}\right)$ & $-0.014\left(\frac{\sqrt{2}}{2} \mu_{8}\right)$ \\
$|001\rangle \rightarrow|200\rangle$ & 3 & 0.01 & $0.442\left(\mu_{7}\right)$ & $0.013\left(\frac{\sqrt{2}}{2} \mu_{7}\right)$ \\
$|101\rangle \rightarrow|300\rangle$ & 3 & 0.02 & $0.034\left(\mu_{7}^{\prime}\right)$ & $0.023\left(\frac{\sqrt{6}}{2} \mu_{7}\right)$ \\
\hline$|001\rangle \rightarrow|300\rangle$ & 4 & - & $0.023\left(\mu_{11}\right)$ & $0.000\left(\frac{\sqrt{6}}{6} \mu_{11}\right)$ \\
$|200\rangle \rightarrow|002\rangle$ & 4 & - & $0.092\left(\mu_{12}\right)$ & $-0.005\left(\frac{1}{2} \mu_{12}\right)$ \\
\hline \hline
\end{tabular}

Table 2: Hamiltonian and transition polarizability parameters of the RDM model.

\begin{tabular}{cc|cc}
\hline \hline$\hbar \omega_{1}$ & 4.7 (fixed) & $\hbar \omega_{3}$ & 6.6 (fixed) \\
$\Delta_{1} / \hbar \omega_{1}$ & $-0.52 \%$ & $\Delta_{3} / \hbar \omega_{3}$ & $-0.10 \%$ \\
$\beta_{12} / \hbar \omega_{1}$ & $-0.05 \%$ & $\beta_{13} / \hbar \omega_{3}$ & $+0.14 \%$ \\
\hline$\alpha_{1}$ & 1.00 (fixed) & $\alpha_{2}$ & -0.003 \\
\hline \hline
\end{tabular}

mal modes, their mechanical coupling $\beta_{i j}$, and the first order polarizability elements $\alpha_{1}$ and $\alpha_{3}$. We find small anharmonicities and mechanical coupling elements, which points to relatively harmonic, weakly coupled normal modes in bromoform. This conclusion is in agreement with vibrational second-order perturbation theory (VPT2) calculations performed previously. ${ }^{31}$ Finally, the polarizability matrix elements obtained in this current work are in good agreement with those obtained in our previous work, ${ }^{31}$ with very small nonlinear elements indicating that the final Raman interaction is indeed a linear process.

It is worth noting that the simulated spectrum has low-intensity peaks at $f_{2} \simeq 6.6 \mathrm{THz}$ that bear resemblance to those in the experimental spectrum. Given weakness of these peaks, they make a small contribution to the error function in the fitting and are thus poorly resolved. Additionally, in some fits, we found that the features at $f_{2} \simeq 5.5 \mathrm{THz}$ might be connected to the mechanical coupling between $Q_{1,2}$ and $Q_{3}$. Future refinement of the RDM model and higher resolution experiments are needed to interpret these subtle features.

\section{Conclusions}

Our original TTR study of bromoform ${ }^{31}$ revealed large dipole nonlinearities which were inconsistent with $a b$ initio electronic structure calculations. Here, we refine both the experimental and theoretical description of this system, resolving the inconsistency. The key refinements are described below.

First, we revised the experimental spectrum by introducing a simplifying time-coordinate transformation. Due to the symmetry of the experimental setup, the time response must be skewed and flipped when the ordering of the two $\mathrm{THz}$ pulses is changed before taking the Fourier transform for a correct interpretation. The new spectrum reveals fewer features and true rephasing signals which are symmetric with their non-rephasing counterparts, as expected.

Second, we have developed an RDM model that analytically includes all dipole and polarizability matrix elements up to $4^{\text {th }}$ order. We also include mechanical anharmonicity and mode- 
coupling in the model Hamiltonian. This refined RDM model preserves the symmetry of the bromoform molecule.

Lastly, we rigorously account for the inversion symmetry of the liquid by excluding $4^{\text {th }}$ order field interactions from the response. We achieve this by simulating the response of the system to both positive and negative $\mathrm{THz}$ fields and combining the two results. The final response is cubic in the field, in agreement with the experimentally measured TTR response.

The revised experimental spectrum is used to fit the parameters of the updated RDM model, leading to good agreement. The fitted nonlinearities are orders of magnitude smaller than found in our previous work and agree well with $a b$ initio electronic structure calculations. Regardless, the original conclusion that nonlinearities in the dipole surface of the intramolecular vibrations drive the TTR response of bromoform remains unchanged.

\section{Acknowledgements}

The authors thank Ralph Welsch, Ian Finneran, Matthew Welborn, and Philip Shushkov for helpful discussions, as well as Peter Hamm for a sharing a copy of their forthcoming manuscript. This work was supported by the National Science Foundation (Grant CHE-1665467) and the Office of Naval Research (Grant N00014-10-10884).

\section{References}

(1) Fecko, C. J.; Eaves, J. D.; Loparo, J. J.; Tokmakoff, A.; Geissler, P. L. Ultrafast hydrogen-bond dynamics in the infrared spectroscopy of water. Science 2003, 301, 1698-1702.

(2) Zheng, J.; Kwak, K.; Fayer, M. D. Ultrafast 2D IR vibrational echo spectroscopy. Acc. Chem. Res. 2007, 40, 75-83.

(3) Park, S.; Fayer, M. D. Hydrogen bond dynamics in aqueous $\mathrm{NaBr}$ solutions. Proc. Natl. Acad. Sci. U. S. A. 2007, 104, 16731-16738.
(4) Ramasesha, K.; De Marco, L.; Mandal, A.; Tokmakoff, A. Water vibrations have strongly mixed intra-and intermolecular character. Nat. Chem. 2013, 5, 935.

(5) Thämer, M.; De Marco, L.; Ramasesha, K.; Mandal, A.; Tokmakoff, A. Ultrafast 2D IR spectroscopy of the excess proton in liquid water. Science 2015, 350, 78-82.

(6) Dahms, F.; Fingerhut, B. P.; Nibbering, E. T.; Pines, E.; Elsaesser, T. Largeamplitude transfer motion of hydrated excess protons mapped by ultrafast 2D IR spectroscopy. Science 2017, 357, 491-495.

(7) Shim, S.-H.; Gupta, R.; Ling, Y. L.; Strasfeld, D. B.; Raleigh, D. P.; Zanni, M. T. Two-dimensional IR spectroscopy and isotope labeling defines the pathway of amyloid formation with residue-specific resolution. Proc. Natl. Acad. Sci. U. S. A. 2009, 106, 6614-6619.

(8) Bagchi, S.; Boxer, S. G.; Fayer, M. D. Ribonuclease $\mathrm{S}$ dynamics measured using a nitrile label with 2D IR vibrational echo spectroscopy. J. Phys. Chem. B 2012, 116, 4034-4042.

(9) Kratochvil, H. T.; Carr, J. K.; Matulef, K.; Annen, A. W.; Li, H.; Maj, M.; Ostmeyer, J.; Serrano, A. L.; Raghuraman, H.; Moran, S. D. et al. Instantaneous ion configurations in the $\mathrm{K}+$ ion channel selectivity filter revealed by 2D IR spectroscopy. Science 2016, 353, 1040-1044.

(10) Heugen, U.; Schwaab, G.; Bründermann, E.; Heyden, M.; Yu, X.; Leitner, D.; Havenith, M. Solute-induced retardation of water dynamics probed directly by terahertz spectroscopy. Proc. Natl. Acad. Sci. U. S. A. 2006, 103, 12301-12306.

(11) Heisler, I. A.; Mazur, K.; Meech, S. R. Low-frequency modes of aqueous alkali halide solutions: An ultrafast optical Kerr effect study. J. Phys. Chem. B 2011, 115, 1863-1873. 
(12) Shalit, A.; Ahmed, S.; Savolainen, J.; Hamm, P. Terahertz echoes reveal the inhomogeneity of aqueous salt solutions. Nat. Chem. 2017, 9, 273.

(13) Xu, J.; Plaxco, K. W.; Allen, S. J. Probing the collective vibrational dynamics of a protein in liquid water by terahertz absorption spectroscopy. Protein Sci. 2006, 15, 1175-1181.

(14) González-Jiménez, M.; Ramakrishnan, G.; Harwood, T.; Lapthorn, A. J.; Kelly, S. M.; Ellis, E.; Wynne, K. Observation of coherent delocalised phonon-like modes in DNA under physiological conditions. Nat. Commun. 2016, 7, 11799 .

(15) Turton, D. A.; Senn, H. M.; Harwood, T.; Lapthorn, A. J.; Ellis, E. M.; Wynne, K. Terahertz underdamped vibrational motion governs protein-ligand binding in solution. Nat. Commun. 2014, 5, 3999.

(16) Tokmakoff, A.; Lang, M.; Larsen, D.; Fleming, G.; Chernyak, V.; Mukamel, S. Two-Dimensional Raman Spectroscopy of Vibrational Interactions in Liquids. Phys. Rev. Lett. 1997, 79, 2702-2705.

(17) Blank, D. A.; Kaufman, L. J.; Fleming, G. R. Fifth-order two-dimensional Raman spectra of CS 2 are dominated by third-order cascades. J. Chem. Phys. 1999, 111, 3105-3114.

(18) Kaufman, L. J.; Heo, J.; Ziegler, L. D.; Fleming, G. R. Heterodyne-detected fifthorder nonresonant Raman scattering from room temperature CS 2. Phys. Rev. Lett. 2002, 88, 207402.

(19) Kubarych, K. J.; Milne, C. J.; Lin, S.; Astinov, V.; Miller, R. J. D. Diffractive optics-based six-wave mixing: Heterodyne detection of the full chi((5)) tensor of liquid CS2. J. Chem. Phys. 2002, 116, 20162042 .

(20) Marder, S. R.; Perry, J. W.; Schaefer, W. P. Synthesis of organic salts with large second-order optical nonlinearities. Science 1989, 245, 626-628.

(21) Schneider, A.; Neis, M.; Stillhart, M.; Ruiz, B.; Khan, R. U. a.; Günter, P. Generation of terahertz pulses through optical rectification in organic DAST crystals: theory and experiment. J. Opt. Soc. Am. $B$ 2006, 23, 1822.

(22) Lu, J.; Li, X.; Zhang, Y.; Hwang, H. Y.; Ofori-Okai, B. K.; Nelson, K. A. Multidimensional Time-Resolved Spectroscopy; Springer, 2019; pp 275-320.

(23) Savolainen, J.; Ahmed, S.; Hamm, P. Two-dimensional Raman-terahertz spectroscopy of water. Proc. Natl. Acad. Sci. U. S. A. 2013, 110, 20402-20407.

(24) Hamm, P.; Shalit, A. Perspective: Echoes in 2D-Raman-THz spectroscopy. J. Chem. Phys. 2017, 146, 130901.

(25) Berger, A.; Ciardi, G.; Sidler, D.; Hamm, P.; Shalit, A. Impact of nuclear quantum effects on the structural inhomogeneity of liquid water. Proc. Natl. Acad. Sci. U. S. A. 2019, 116, 2458-2463.

(26) Hoffmann, M. C.; Brandt, N. C.; Hwang, H. Y.; Yeh, K. L.; Nelson, K. A. Terahertz Kerr effect. Appl. Phys. Lett. 2009, 95, 231105.

(27) Allodi, M. A.; Finneran, I. A.; Blake, G. A. Nonlinear terahertz coherent excitation of vibrational modes of liquids. J. Chem. Phys. 2015, 143, 234204 .

(28) Sajadi, M.; Wolf, M.; Kampfrath, T. Transient birefringence of liquids induced by terahertz electric-field torque on permanent molecular dipoles. Nat. Commun. 2017, 8, 14963 .

(29) Zalden, P.; Song, L.; Wu, X.; Huang, H.; Ahr, F.; Mücke, O. D.; Reichert, J.; Thorwart, M.; Mishra, P. K.; Welsch, R. et al. Molecular polarizability anisotropy 
of liquid water revealed by terahertzinduced transient orientation. Nat. Commun. 2018, 9, 2142.

(30) Finneran, I. A.; Welsch, R.; Allodi, M. A.; Miller, T. F.; Blake, G. A. Coherent two-dimensional terahertz-terahertzRaman spectroscopy. Proc. Natl. Acad. Sci. U. S. A. 2016, 113, 6857-6861.

(31) Finneran, I. A.; Welsch, R.; Allodi, M. A.; Miller, T. F.; Blake, G. A. 2D THzTHz-Raman Photon-Echo Spectroscopy of Molecular Vibrations in Liquid Bromoform. J. Phys. Chem. Lett. 2017, 8, 46404644.

(32) Ciardi, G.; Berger, A.; Hamm, P.; Shalit, A. Signatures of Intra/Intermolecular Vibrational Coupling in Halogenated Liquids Revealed by 2D Raman-THz Spectroscopy. J. Phys. Chem. Lett. 2019, in press, PMID: 31318212.

(33) Teixeira-Dias, J. The Raman spectra of bromoform. Spectrochim. Acta, Part A 1979, 35, 857-859.

(34) Steffen, T.; Fourkas, J. T.; Duppen, K. Time resolved four-and six-wave mixing in liquids. I. Theory. J. Chem. Phys. 1996, 105, 7364-7382.

(35) Steffen, T.; Duppen, K. Population relaxation and non-Markovian frequency fluctuations in third-and fifth-order Raman scattering. Chem. Phys. 1998, 233, 267285.

(36) Okumura, K.; Tanimura, Y. Energy-level diagrams and their contribution to fifthorder Raman and second-order infrared responses: Distinction between relaxation models by two-dimensional spectroscopy. J. Phys. Chem. A 2003, 107, 8092-8105.

(37) Hasegawa, T.; Tanimura, Y. Calculating fifth-order Raman signals for various molecular liquids by equilibrium and nonequilibrium hybrid molecular dynamics simulation algorithms. J. Chem. Phys. 2006, 125, 74512 .

(38) Tanimura, Y.; Ishizaki, A. Modeling, Calculating, and Analyzing Multidimensional Vibrational Spectroscopies. Acc. Chem. Res. 2009, 42, 1270-1279.

(39) Hamm, P.; Savolainen, J. Twodimensional-Raman-terahertz spectroscopy of water: Theory. J. Chem. Phys. 2012, 136, 94516.

(40) Hamm, P.; Savolainen, J.; Ono, J.; Tanimura, Y. Note: Inverted time-ordering in two-dimensional-Raman-terahertz spectroscopy of water. J. Chem. Phys. 2012, 136, 236101.

(41) Hamm, P. 2D-Raman-THz spectroscopy: A sensitive test of polarizable water models. J. Chem. Phys. 2014, 141, 184201.

(42) Ito, H.; Hasegawa, T.; Tanimura, Y. Calculating two-dimensional THz-Raman$\mathrm{THz}$ and Raman-THz-THz signals for various molecular liquids: The samplers. $J$. Chem. Phys. 2014, 141, 124503.

(43) Ito, H.; Jo, J. Y.; Tanimura, Y. Notes on simulating two-dimensional Raman and terahertz-Raman signals with a full molecular dynamics simulation approach. Struct. Dyn. 2015, 2, 54102.

(44) Ikeda, T.; Ito, H.; Tanimura, Y. Analysis of 2D THz-Raman spectroscopy using a non-Markovian Brownian oscillator model with nonlinear system-bath interactions. J. Chem. Phys. 2015, 142, 212421.

(45) Jo, J. Y.; Ito, H.; Tanimura, Y. Full molecular dynamics simulations of liquid water and carbon tetrachloride for twodimensional Raman spectroscopy in the frequency domain. Chem. Phys. 2016, 481, 245-249.

(46) Ito, H.; Hasegawa, T.; Tanimura, Y. Effects of Intermolecular Charge Transfer in Liquid Water on Raman Spectra. J. Phys. Chem. Lett. 2016, 7, 4147-4151. 
(47) Ito, H.; Tanimura, Y. Simulating twodimensional infrared-Raman and Raman spectroscopies for intermolecular and intramolecular modes of liquid water. $J$. Chem. Phys. 2016, 144, 74201.

(48) Sidler, D.; Hamm, P. Feynman diagram description of 2D-Raman-THz spectroscopy applied to water. J. Chem. Phys. 2019, 150, 044202.

(49) Hamm, P.; Zanni, M. Concepts and methods of 2D infrared spectroscopy; Cambridge University Press, 2011.

(50) Steffen, T.; Tanimura, Y. Twodimensional spectroscopy for harmonic vibrational modes with nonlinear systembath interactions. I. Gaussian-white case. J. Phys. Soc. Jpn. 2000, 69, 3115-3132.

(51) Tanimura, Y.; Steffen, T. Twodimensional spectroscopy for harmonic vibrational modes with nonlinear systembath interactions. II. Gaussian-Markovian case. J. Phys. Soc. Jpn. 2000, 69, 4095-4106.

(52) Ishizaki, A.; Tanimura, Y. Modeling vibrational dephasing and energy relaxation of intramolecular anharmonic modes for multidimensional infrared spectroscopies. J. Chem. Phys. 2006, 125, 084501.

(53) Fernández-Liencres, M. P.; Navarro, A.; López, J. J.; Fernández, M.; Szalay, V.; de los Arcos, T.; Garcia-Ramos, J. V.; Escribano, R. M. The force field of bromoform: A theoretical and experimental investigation. J. Phys. Chem. 1996, 100, 16058-16065.

(54) Levenberg, K. A method for the solution of certain non-linear problems in least squares. Q. Appl. Math. 1944, 2, 164-168.

(55) Marquardt, D. W. An algorithm for leastsquares estimation of nonlinear parameters. J. Soc. Ind. Appl. Math. 1963, 11, 431-441.
(56) Eaton, J. W.; Bateman, D.; Hauberg, S.; Wehbring, R. GNU Octave version 4.2.0 manual: a high-level interactive language for numerical computations. 2016. 


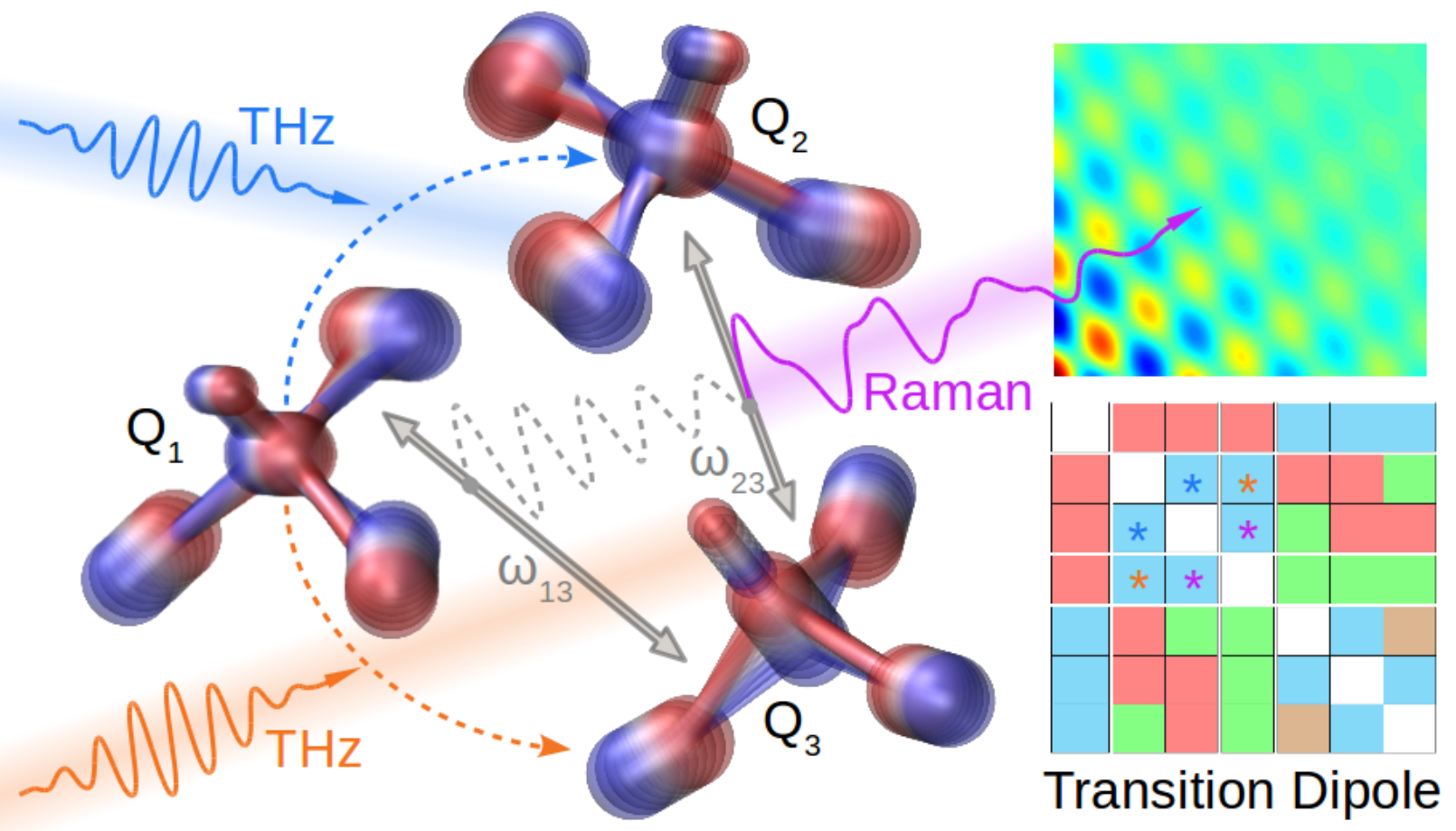

Figure 6: TOC figure. 